\title{
Anti-Obesity, Anti-Atherosclerotic and Anti-Oxidant Effects of Pu-Erh Tea on a High Fat Diet-Induced Obese Rat Model
}

\author{
Changyun Xiong1*, Yuanju Peng1, Benying Liu ${ }^{2}$, Wenrui Cui ${ }^{1}, X^{1}$ cheng Liu ${ }^{3}$ \\ ${ }^{1}$ College of Tropical Crops, Yunnan Agricultural University, Pu'er, China \\ ${ }^{2}$ Tea Research Institute, Yunnan Academy of Agricultural Science, Menghai, China \\ ${ }^{3}$ South China Botanical Garden, Chinese Academy of Sciences, Guangzhou, China \\ Email: *spandax@163.com
}

How to cite this paper: Xiong, C.Y., Peng, Y.J., Liu, B.Y., Cui, W.R. and Liu, X.C. (2019) Anti-Obesity, Anti-Atherosclerotic and Anti-Oxidant Effects of Pu-Erh Tea on a High Fat Diet-Induced Obese Rat Model. Journal of Biosciences and Medicines, 7 , 120-130.

https://doi.org/10.4236/jbm.2019.72010

Received: January 9, 2019

Accepted: February 24, 2019

Published: February 27, 2019

Copyright $\odot 2019$ by author(s) and Scientific Research Publishing Inc. This work is licensed under the Creative Commons Attribution International License (CC BY 4.0).

http://creativecommons.org/licenses/by/4.0/

\begin{abstract}
$\mathrm{Pu}$-erh tea, a traditional Chinese beverage, has been believed to have many benefits to human health and without side effects. In this study, we systematically analyzed the main active components of Pu-erh tea and investigated its anti-obesity, anti-atherosclerotic and anti-oxidant effects using an obese rat model. Obesity was induced by feeding a high-fat diet and subsequently the experimental obese mice were fed with high-fat diet supplemented with low (2.5\%), medium (5\%) or high (7.5\%) doses of Pu-erh tea powder for 6 weeks respectively. As result, the body weight gain of the rats was decreased by medium and high doses of $\mathrm{Pu}$-erh tea treatments. Furthermore, the levels of serum total cholesterol (TC), triglyceride (TG) and atherosclerosis index (AI) were significantly lowered by $\mathrm{Pu}$-erh tea compared to the control group. Conversely, high density lipoprotein-cholesterol (HDL-C) level of the rats was significantly elevated by Pu-erh tea treatments. In addition, Pu-erh tea treatments increased the activities of anti-oxidative enzymes such as superoxide dismutase (SOD) and glutathione peroxides (GSH-Px), whereas reduced the level of lipid peroxidation product malondialdehyde (MDA) in obese rats. Collectively, our findings revealed that $\mathrm{Pu}$-erh tea exerts comprehensive benefits in anti-obesity, anti-atherosclerotic and anti-oxidant effects, therefore can be used as a promising functional food in obesity management.
\end{abstract}

\section{Keywords}

Pu-Erh Tea, Anti-Obesity, Anti-Atherosclerotic, Anti-Oxidant, Obese Rat Model 


\section{Introduction}

Obesity is a chronic metabolic disorder that results from the imbalance between energy intake and energy expenditure, it is characterized by enlarged fat mass and elevated lipid concentration in blood [1]. Now obesity is becoming a well-known risk factor for variety of life-style diseases, such as hyperlipidemia, hypertension, and diabetes all over the world, especially in the developed and most developing countries. Over-accumulation of lipid leads to the formation of atherosclerotic plaques, which could either grow towards the lumen of the artery or become unstable and rupture, causing an obstruction of the blood flow [2]. Atherosclerotic plaques have become the first threat to human health. Furthermore, obesity is accompanied by the formation of oxygen free radicals, whose intensified activity without effective defense mechanisms can lead to oxidative stress and related complication [3]. Great efforts have been made to find natural foods to prevent obesity and atherosclerosis in the pass decades.

Tea [Camellia sinensis (L.) O. Kuntze], one of the most popular beverages worldwide, has been considered to be a crude medicine for 4000 years. Tea is categorized into three types according to the manufacturing processes, non-fermented tea (green tea), semi-fermented tea (oolong tea), and completely fermented tea (black tea) [4]. As a famous traditional black tea mainly produced in Yunnan province, China, Pu-erh tea exhibits many biological benefits [5]. For example, the powder and extracts of Pu-erh tea demonstrate an anti-oxidant activity both in vitro and in vivo [6], and display a preventive effect on cisplat in-induced liver oxidative damage [7]. Pu-erh tea extracts can restrain the growth of bacterium strains such as Escherichia coli, Bacillus subtilis and Staphylococcus aureus [8]. Furthermore, it was also reported that $\mathrm{Pu}$-erh tea extracts inhibit tumor cell growth [9]. Most importantly, a series of studies suggested that Pu-erh tea can reduce the body weight, suppress hyperlipidemia, reduce the low-density lipoprotein-cholesterol level, and decrease atherogenic index in mammalians [10] [11]. It was reported that theabrownins, one of the main bioactive components in Pu-erh tea, could regulate the activity of key enzymes involved in lipid metabolism and accelerate the catabolism of exogenous cholesterol in rats [12]. Above studies have proposed possible roles of $\mathrm{Pu}$-erh tea in reducing obesity and atherosclerosis risk. However, the association of the main active constituents of $\mathrm{Pu}$-erh tea with its complex roles on anti-obesity, anti-atherosclerotic and anti-oxidant effects in animal model, had not yet been systematically studied.

In the present study, we analyzed the main active constituents of Pu-erh tea and investigated its effects on anti-obesity, anti-atherosclerotic and anti-oxidant using a high-fat diet-induced obese rat model. Our work displayed that Pu-erh tea is rich in many active constituents, such as tea polyphenols, theaflavins, thearubigins, theabrownins, gallic acid and caffeine, which may exert comprehensive benefits on anti-obesity, anti-atherosclerotic and anti-oxidant effects. Our studies revealed that $\mathrm{Pu}$-erh tea can be used as a promising functional food in obesity management. 


\section{Materials and Methods}

\subsection{Materials}

Pu-erh tea was provided by China Academy of Pu-erh Tea (Pu'er, China). Male Sprague Dawley (SD) rats, standard M02-F granule diet and M04-Fgranule diet as high-fat diets were all purchased from Shanghai SLAC laboratory animal Co., LTD., (Shanghai, China) [M02-F (w/w): supplemented with barley flour (20\%), bean flour $(10 \%)$, cornflour (16\%), bran $(16 \%)$, yeast (1\%), fish meal $(10 \%)$, bone meal (5\%), salt (2\%), alfalfa meal (20\%); M04-F (w/w): supplemented with M02-F (80\%), lard (10\%), yolk powder (10\%)]. All reagents and chemicals were of analytical grade.

\subsection{Animals}

Sixty male SD rats were all individually housed in stainless steel wire-bottomed cages and acclimated to the air-conditioned room $\left(23^{\circ} \mathrm{C} \pm 2^{\circ} \mathrm{C}\right.$, humidity $55 \% \pm$ $5 \%, 12 \mathrm{~h} \mathrm{light/dark}$ cycle). All rats were fed M02-F diet and allowed free access to water for one week before the study as described below, the weights of rats at the beginning of the study ranged from 80 to $100 \mathrm{~g}$. The present study was approved by the Institutional Animal Care and Use Committee of Zhejiang University. All applicable institutional and governmental regulations concerning the ethical use of animals were followed during this research.

\subsection{Determination of Principal Components in Pu-Erh Tea Powder}

$\mathrm{Pu}$-erh tea powder was prepared through grinding and sieving with 40-mesh sieve. Water and water extract contents were determined according to the national standards in China. Levels of theanine were determined by a reverse phase high-performance liquid chromatography (HPLC) system coupled with a detector at $425 \mathrm{~nm}$ absorbance [13]. The ferrous tartrate colorimetric method was used to detect the total contents of tea polyphenol [14]. Caffeine and the composition of tea polyphenol were analyzed by using another HPLC method as described previously [15]. TF (theaflavins), TR (thearubigins) and TB (theabrownins) were separated by ethyl acetate and n-butyl alcohol, their contents were quantitated using the spectrophotometry method.

\subsection{Anti-Obesity and Anti-Atherosclerotic Test of Pu-Erh Tea in Obese Rats}

After one week of acclimation, the rats were randomly divided into two groups according the body weight: the control group (CG, 10 rats) was fed M02-F diet while the other 50 rats were fed M04-F diet. After 30 days, animals fed the high-fat diet were measured the body weight, all of these rats met the obesity model criteria (when their body weight reached $10 \%$ more than control group) and were, thus, enrolled into the experiment by randomly dividing into 5 groups of 10 rats each. One served as obesity model group (MG), giving M04-F diet; one 
acted as diet control group (DCG), giving M02-F diet, the rest 3 group as low dose group (LDG), middle dose group (MDG) and high dose group (HDG), which were given M04-F diet with 2.5\%, 5\%, 7.5\% Pu-erh tea powder respectively. The rats had free access to food and water, and their food consumption was measured every other day while their body weight was measured weekly. After 6 weeks, the rats were anesthetized with sodium pentobarbital after fasting for $12 \mathrm{~h}$. Blood samples were taken from the inferior venacava to determine the plasma biomarkers. After collecting the blood, the liver and white adipose tissues (epididymal and prerenal) were removed, rinsed with a physiological saline solution and weighed immediately. The serum, liver and adipose tissues were prepared and frozen at $-20^{\circ} \mathrm{C}$ until analyzed.

\subsection{Assay of Blood Lipids and Lipoproteins}

Serum levels of total cholesterol (TC), triglycerides (TG), and high density lipoprotein-cholesterol (HDL-C) were measured using reagent kits method (Nanjing Jiancheng Bioengineering Institute, Nanjing, china). The atherosclerotic index (AI) was calculated using the formula:

$$
\mathrm{AI}=(\mathrm{TC}-\mathrm{HDL}-\mathrm{C}) / \mathrm{HDL}-\mathrm{C}
$$

\subsection{Examination of Antioxidant Enzyme Activity}

The activities of anti-oxidant enzymes such as superoxide dismutase (SOD) and glutathione peroxides (GSH-Px), and lipid peroxidation product malondialdehyde (MDA) were determined using reagent kits method (Nanjing Jiancheng Bioengineering Institute, Nanjing, China).

\subsection{Statistical Analysis}

All data were recorded as means \pm standard error (S.E.) from 10 rats in each group. Statistical analysis was performed by SPSS17.0 with one-way ANOVA procedures. Significant differences between means were determined by Duncan's multiple range tests at the level of $\mathrm{p}<0.05$ or $\mathrm{p}<0.01$.

\section{Results and Discussion}

\subsection{The Main Constituents of Pu-Erh Tea Powder}

To investigate the physiological effects of $\mathrm{Pu}$-erh tea, we firstly examined the main constituents of $\mathrm{Pu}$-erh tea powder and its raw material, Shai Qing Mao Cha. As shown in Table 1, the main constituents of $\mathrm{Pu}$-erh tea powder includes water, water extract, tea polyphenols (TP), theaflavins (TF), thearubigins (TR) and theabrownins (TB). Compared to Shai Qing Mao Cha, the contents of TP, TF and TR in Pu-erh tea powder were descend while the TB content was elevated.

We further detected the contents of gallic acid, caffeine, and the principle compositions of tea polyphenols in Pu-erh tea and its raw material. As shown in Table 2, the principle compositions of tea polyphenols in Pu-erh tea included 
Table 1. The main constituents of Pu-erh tea and its raw material.

\begin{tabular}{ccccccc}
\hline Sample & $\begin{array}{c}\text { Water } \\
(\%)\end{array}$ & $\begin{array}{c}\text { Water extract } \\
(\%)\end{array}$ & $\begin{array}{c}\text { Tea polyphenols } \\
(\%)\end{array}$ & TF $(\%)$ & TR (\%) & TB (\%) \\
\hline Pu-erh tea & $9.04 \pm 0.33$ & $30.14 \pm 0.86$ & $12.80 \pm 0.88$ & $0.16 \pm 0.22$ & $0.34 \pm 0.26$ & $11.35 \pm 0.26$ \\
Raw materials & $6.58 \pm 0.21$ & $43.76 \pm 0.45$ & $33.88 \pm 1.02$ & $0.25 \pm 0.16$ & $6.43 \pm 0.03$ & $2.6 \pm 0.03$ \\
\hline
\end{tabular}

Notes: all data are presented as mean \pm SEM.

Table 2. The contents of gallic acid, caffeine, and the principal compositions of tea polyphenols in Pu-erh tea and its raw material (mg/g).

\begin{tabular}{cccccccc}
\hline Sample & gallic acid & caffeine & EGC & EC & EGCG & GCG & ECG \\
\hline Pu-erh tea & 0.671 & 2.823 & 0.11 & 0.08 & - & - & - \\
Raw material & - & 2.654 & 2.03 & 2.10 & 7.15 & 1.190 & 14.66 \\
\hline
\end{tabular}

Notes: All data are presented as mean \pm SEM.

(-)-epigallocatechin (EGC) and (-)-epicatechin (EC). Compared to raw materials, the contents of EGC and EC was largely descend, and the contents of (-)-epigallocatechin gallate (EGCG), (-)-gallocatech in gallate (GCG) and (-)-epicatechin gallate (ECG) were even not detected in Pu-erh tea powder. In contrast, the content of gallic acid was significantly elevated, whereas the content of caffeine was not significantly changed in Pu-erh tea powder compared to raw materials (Table 2). Collectively, these data revealed that Pu-erh tea is rich in many active constituents, including TF, TB, TR, gallic acid, caffeine and TP (EGC and EC), which may exert multiple physiological benefits to improve human health.

\subsection{Effect of Pu-Erh Tea on the Body Weight Gain of Obese Rats}

To investigate effect of Pu-erh tea on body weight gain of obese rats, the model obese rats were constructed ant then fed high fat diet supplemented with low, medium and high doses of Pu-erh tea powder, respectively as described in Materials and Methods. As shown in Figure 1, as expected, the body weight of obesity model group (MG) was significantly higher than the control group (CG) $(\mathrm{p}<$ 0.01 ), while the body weight of diet control group (DCG) decreased significantly compared to the obesity model group $(M G)(p<0.05)$. For the Pu-erh tea treated groups (PEG), low dose group showed no significant effect on the body weight compared to the obesity model group, however, the medium and high dose groups exhibited a remarkably reduced body weight compared to the obesity model group $(\mathrm{p}<0.05)$ (Figure 1). Moreover, it was interested to find that there was no significant difference in total food intake among the groups with or without $\mathrm{Pu}$-erh tea treatments (Figure 1), indicating that body weight reduction of obese rats was independently of food intake. Collectively, these findings revealed that medium and high doses of Pu-erh tea treatments effectively lower body weight gain of obese rats. 




Figure 1. Effects of Pu-erh tea on body weight and food intake of rats fed either normal or high fat diets, all data are presented as mean \pm SEM. ${ }^{*} \mathrm{p}<0.05,{ }^{* *} \mathrm{p}<0.01$; CG, control group; MG, obesity model group; DCG, diet control group; PEG, Pu-erh tea treated group, low, middle and high dose group from left to right.

\subsection{Effect of Pu-Erh Tea on the Serum Levels of TC and TG in Obese Rats}

We further measured the levels of TC and TG, two important indexes closely related to obesity, in rats fed Pu-erh tea. As shown in Figure 2, the serum levels of TC and TG in obesity model group were both significantly higher than those in the control group $(p<0.01)$, confirming that high fatty diet can induce the levels of TC and TG. We further showed that the levels of TC in Pu-erh tea treated groups were significant lower than the obesity model group $(\mathrm{p}<0.05)$. A significant reduction in TG levels by $20.10 \%$ and $25.62 \%$ was detected in the medium and high dose groups compared with the obesity model group, respectively (Figure 2(b)). These findings therefore indicated that medium and high doses of $\mathrm{Pu}$-erh tea can reduce the levels of serum TC and TG, which is consistent with its effect on the body weight of obese rats.

\subsection{Anti-Atherosclerotic Effect of Pu-Erh Tea on Obese Rats}

The benefit of $\mathrm{Pu}$-erh tea reducing body weight prompted us to detect its anti-atherosclerotic effect. The level of HDL-C (high density lipoprotein-cholesterol), a primary component of TC in the serum, which regarding as "good cholesterol" in animal body was measured on obese rats after fed Pu-erh tea. It was demonstrated that either Pu-erh tea treatments or diet control group increased serum levels of HDL-C in obese rats. After treated with $2.5 \%, 5 \%$ and $7.5 \%$ of Pu-erh tea for 6 weeks, serum levels of HDL-C increased by 27.59\%, 43.68\% and 62.07\%, respectively (Figure 3(a)), indicating that Pu-erh tea increases the HDL-C level of obese rats in a dose dependent manner. On the contrary, the level of LDL-C (low density lipoprotein-cholesterol), which regarding as "bad cholesterol" in animal body, has been less affected by either Pu-erh tea treatments or diet control group in obese rats.

The atherosclerotic index (AI) is formulated by international medical to measure the atherosclerotic degree. We then compared the AI values among obesity model group, diet control group and $\mathrm{Pu}$-erh tea treated groups. As shown in Figure $3(\mathrm{~b})$, both diet control and $\mathrm{Pu}$-erh tea treatments significantly 


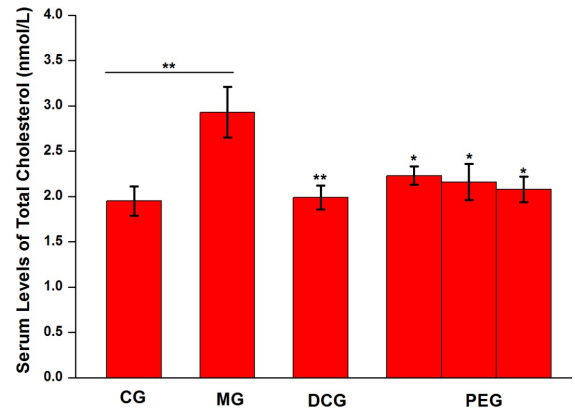

(a)

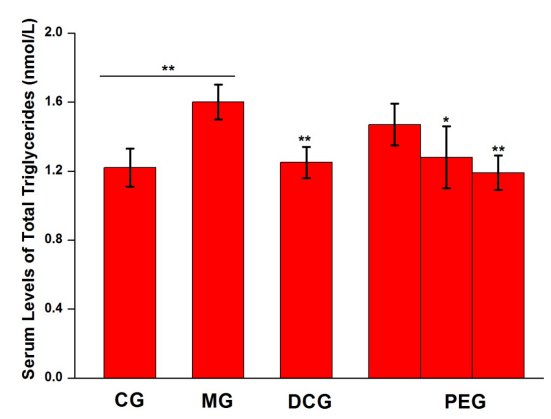

(b)

Figure 2. Effects of Pu-erh tea on serum levels of total cholesterol (a) and triglycerides (b) in obese rats, all data are presented as mean \pm SEM. ${ }^{\star} \mathrm{p}<0.05,{ }^{* *} \mathrm{p}<0.01$; CG, control group; MG, obesity model group; DCG, diet control group; PEG, Pu-erh tea treated group, low, middle and high dose group from left to right.

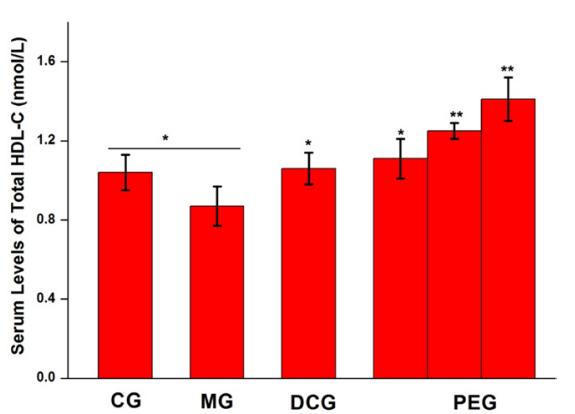

(a)



(b)

Figure 3. Effects of Pu-erh tea on serum levels of HDL-C (a); LDL-C (b) and AI (c) in obese rats, all data are presented as mean \pm SEM. ${ }^{*} \mathrm{p}<0.05,{ }^{* *} \mathrm{p}<0.01$; CG, control group; MG, obesity model group; DCG, diet control group; PEG, Pu-erh tea treated group, low, middle and high dose group from left to right.

decreased the AI values in the obese rats $(\mathrm{p}<0.01)$. Under low, medium and high doses Pu-erh tea treatments, the AI values were declined 57.38\%, 69.20\% and $79.75 \%$ compared to control group, respectively (Figure $3(\mathrm{~b})$ ). Together, these data indicated that $\mathrm{Pu}$-erh tea exerts a significant function to reduce the risk of atherosclerosis.

\subsection{Antioxidant Effect of Pu-Erh Tea on Obese Rats}

We further detected the antioxidant activity of the serum in rats fed $\mathrm{Pu}$-erh tea. Previous studies have reported that oxidative injuries induced by excessively produced free radicals are protected by scavenging enzymes such as superoxide dismutase (SOD) and glutathione peroxides (GSH-Px) in mammalians [16]. Malondialdehyde (MDA), the lipid peroxidation product, has been widely used as a biomarker of oxidative stress. As shown in Table 3, the activities of SOD and GSH-Px in high fat diet-induced obesity model rat group were significantly lower whereas the levels of MDA were significantly higher compared to the control group, confirming the negative effect of high fat diet on antioxidant activity of rats. After fed low, medium and high doses of $\mathrm{Pu}$-erh tea, the activities of 
Table 3. Effects of Pu-erh tea on serum anti-oxidation of obese rats.

\begin{tabular}{cccccc}
\hline Group & Diet & Treatment & SOD (U/mL) & GSH-Px $(\mathrm{U} / \mathrm{mL})$ & MDA (mM) \\
\hline Control & M02-F & None & $223.1 \pm 19.16^{* *}$ & $2083.7 \pm 132.4^{* *}$ & $4.09 \pm 0.73^{* *}$ \\
Obesity model & M04-F & None & $160.9 \pm 10.28$ & $1116.6 \pm 178.4$ & $10.53 \pm 1.12$ \\
Diet control & M02-F & None & $218.9 \pm 20.13^{* *}$ & $2004.5 \pm 98.3^{* *}$ & $4.88 \pm 1.04^{\star *}$ \\
Pu-erh tea & M04-F & 2.5\% Pu-erh tea & $178.8 \pm 25.51$ & $1986.4 \pm 123.5^{* *}$ & $5.69 \pm 0.95^{* *}$ \\
Pu-erh tea & M04-F & 5\% Pu-erh tea & $206.1 \pm 28.26^{* *}$ & $2129.4 \pm 192.2^{* *}$ & $4.78 \pm 0.86^{* *}$ \\
Pu-erh tea & M04-F & $7.5 \%$ Pu-erh tea & $212.7 \pm 30.14^{* *}$ & $2164.5 \pm 201.6^{* *}$ & $4.46 \pm 0.74^{* *}$ \\
\hline
\end{tabular}

Notes: All data are presented as mean \pm SEM. ${ }^{*} \mathrm{p}<0.05,{ }^{* *} \mathrm{p}<0.01$; M02-F: Standard granule diet, M04-F: high-fat diet.

SOD were increased by $11.10 \%, 28.02 \%$ and $32.15 \%$, and the levels of GSH-Px were also enhanced by $77.89 \%, 90.70 \%$ and $93.84 \%$ compared to the control group, respectively (Table 3 ). Consistent with changes of antioxidant enzymes, serum levels of MDA were significant decreased in the obese rats treated with Pu-erh tea compared to obesity model (Table 3 ). Taken together, these data revealed that $\mathrm{Pu}$-erh tea could increase the antioxidant activity of the serum in obese rats.

\section{Discussion}

Nowadays, Obesity constitutes a significant public problem which is associated with many risks including the formation of atherosclerotic plaque. Meanwhile, with the obesity problem more and more serious consequences and taking diet pills. Pu-erh tea, a traditional Chinese beverage, a natural product, has been proposed to be beneficial for human health because of its unique lipid-lowering effect and without any toxicity and side effects, which caused the extensive concern of the medical profession and carried on the thorough research. In these studies, we systematically investigated the main active constituents of Pu-erh tea and its anti-obesity, anti-atherosclerotic and anti-oxidant effects on a high fat diet-induced obese rat model. Our findings revealed that Pu-erh tea is rich in active constituents, such as tea polyphenols, theaflavins, thearubigins, theabrownins, gallic acid and caffeine, which exerts comprehensive anti-obesity, anti-atherogenic and anti-oxidative effects in obese rats.

A number of functional compositions were known to be present in teas, including tea polyphenols, theanine, caffeine, tea polysaccharides, vitamin $\mathrm{C}$ and vitamin $\mathrm{E}$, and etc., however, the exact components responsible for its function in anti-obesity keeps unclear. Previous work suggested that the anti-obesity effects of oolong tea in high-fat diet-treated mice might be partly due to the effect of caffeine [17]. It was demonstrated that the caffeine isolated from oolong tea enhanced noradrenaline-induced lipolysis in fat cells and also accelerated the hormone-induced lipolysis [17]. Another work reported that caffeine and theaninein green tea were responsible for the suppressive effects on body weight in- 
crease and fat accumulation, and catechins and caffeine were synergistic in anti-obesity activities [18]. Moreover, it was found that two tea polyphenols, EGCG and theaflavins (TF) suppress body weight by repressing the expression of fatty acid synthase genes [19]. These findings suggested that multiple active constituents in teas may function to reduce the risk of obesity. In present work, a subset of active constituents, including TF, TB, TR, gallic acid, caffeine and TP (EGC and EC) was detected to be abundant in $\mathrm{Pu}$-erh tea powder, which may be responsible for its function on anti-obesity, anti-atherosclerotic effects. $\mathrm{Pu}$-erh tea is post-fermented from Shai Qing Mao Cha, here we showed that the levels of tea polyphenols such as EGC and EC were decreased whereas the level of gallic acids was elevated in Pu-erh tea compared to Shai qing Mao Cha (Table 2), which indicated that the fermentation process may largely convert the active components of $\mathrm{Pu}$-erh tea thus affect its anti-obese and anti-atherogenic activities. It would be interested to analyze the anti-obesity and anti-atherogenic effects of Shai Qing Mao Cha.

Previous studies have separately examined the anti-obesity, anti-atherogenic and anti-oxidant activities of $\mathrm{Pu}$-erh tea extract. It was reported that the $\mathrm{Pu}$-erh tea extract significantly suppresses weight gain of obese rats [20], lowers atherosclerotic risk factors [21], and increases free radicals cavenging activity [6]. It's well known that atherosclerosis is an important complication of obesity [2]. In present work, we systematically analyzed these effects of $\mathrm{Pu}$-erh tea on an obese rat model. We showed that $\mathrm{Pu}$-erh tea lowers body weight gain and the levels of serum TC and TG of obese rats in a dose dependent manner. Reduced body weight usually contributes to reducing the risk of atherosclerosis. Consistently, we found that $\mathrm{Pu}$-erh tea treatments reduce the risk of atherosclerosis by increasing the HDL-C level whereas decrease the atherosclerotic index in obese rats. Furthermore, reactive oxygen species (ROS) have been hypothesized to play causative role in numerous diseases including atherosclerosis [22]. Here we showed that $\mathrm{Pu}$-erh tea treatments increase the activities of anti-oxidative enzymes such as SOD and GSH-Px, and reduce the level of lipid peroxidation product $\mathrm{MDA}$, which indicated that $\mathrm{Pu}$-erh tea may function to eliminate over-accumulated ROS and improve oxidation states of blood lipids in obese rats, which may ultimately reduce the risk of atherosclerotic diseases. Together, these findings proposed that the comprehensive anti-obesity, anti-atherosclerotic and anti-oxidant effects of Pu-erh tea may lie on not only its diverse active constituents but also the interrelationships among the different symptoms.

\section{Conclusion}

In conclusion, our work displayed that $\mathrm{Pu}$-erh tea is rich in many active constituents, which is required for its function in anti-obesity, anti-atherosclerotic and anti-oxidation. Our findings revealed that $\mathrm{Pu}$-erh tea can be used as a promising functional food in obesity management. 


\section{Conflicts of Interest}

The authors declare no conflicts of interest regarding the publication of this paper.

\section{References}

[1] Choi, H., Eo, H., Park, K., Jin, M., Park, E.J., Kim, S.H., Park, J.E. and Kim, S. (2007) A Water-Soluble Extract from Cucurbita moschata Shows Anti-Obesity Effects by Controlling Lipid Metabolism in a High Fat Diet-Induced Obesity Mouse Model. Biochemical and Biophysical Research Communications, 359, 419-425. https://doi.org/10.1016/j.bbrc.2007.05.107

[2] Kolodgie, F.D., Narula, J., Guillo, P. and Virmani, R. (1999) Apoptosis in Human Atherosclerotic Plaques. Apoptosis, 4, 5-10. https://doi.org/10.1023/A:1009645730270

[3] Rowicka, G., Dyląg, H., Ambroszkiewicz, J., Riahi, A., Weker, H. and Chełchowska, M. (2017) Total Oxidant and Antioxidant Status in Prepubertal Children with Obesity. Oxidative Medicine and Cellular Longevity, 2017, Article ID 5621989. https://doi.org/10.1155/2017/5621989

[4] Zeng, L., Yan, J., Luo, L. and Zhang, D. (2015) Effects of Pu-Erh Tea Aqueous Extract (PTAE) on Blood Lipid Metabolism Enzymes. Food \& Function, 6, 2008-2016. https://doi.org/10.1039/C5FO00362H

[5] Lee, L.K. and Foo, K.Y. (2013) Recent Advances on the Beneficial Use and Health Implications of Pu-Erh Tea. Food Research International, 53, 619-628.

https://doi.org/10.1016/j.foodres.2013.02.036

[6] Gong, J.S., Peng, C.X., Xiang, H., Li, J.H., Li, B.C. and Zhou, H.J. (2009) Antioxidant Activity of Extracts of Pu-Erh Tea and Its Material. Asian Journal of Agricultural Sciences, 1, 48-54.

[7] Zheng, X.N., Wang, X.W., Li, L.Y., Xu, Z.W., Huang, H.Y., Zhao, J.S., Zhang, D., Yin, X., Sheng, J. and Tang, J.T. (2014) Pu-Erh Tea Powder Preventive Effects on Cisplatin-Induced Liver Oxidative Damage in Wistar Rats. Asian Pacific Journal of Cancer Prevention, 15, 7389-7394. https://doi.org/10.7314/APJCP.2014.15.17.7389

[8] Wu, S.-C., Yen, G.-C., Wang, B.-S., Chiu, C.-K., Yen, W.-J., Chang, L.-W. and Duh, P.-D. (2007) Antimutagenic and Antimicrobial Activities of Pu-Erh Tea. LWT-Food Science and Technology, 40, 506-512. https://doi.org/10.1016/j.lwt.2005.11.008

[9] Zhao, L., Jia, S., Tang, W., Sheng, J. and Luo, Y. (2011) Pu-ERh Tea Inhibits Tumor Cell Growth by Down-Regulating Mutant p53. International Journal of Molecular Sciences, 12, 7581-7593. https://doi.org/10.3390/ijms12117581

[10] Kuo, K.L., Weng, M.S., Chiang, C.T., Tsai, Y.J., Lin-Shiau, S.Y. and Lin, J.K. (2005) Comparative Studies on the Hypolipidemic and Growth Suppressive Effects of Oolong, Black, Pu-Erh, and Green Tea Leaves in Rats. Journal of Agricultural and Food Chemistry, 53, 480-489. https://doi.org/10.1021/jf049375k

[11] Fujita, H. and Yamagami, T. (2008) Efficacy and Safety of Chinese Black Tea (Pu-Ehr) Extract in Healthy and Hypercholesterolemic Subjects. Annals of Nutrition and Metabolism, 53, 33-42. https://doi.org/10.1159/000153006

[12] Gong, J., Peng, C., Chen, T., Gao, B. and Zhou, H. (2010) Effects of Theabrownin from Pu-Erh Tea on the Metabolism of Serum Lipids in Rats: Mechanism of Action. Journal of Food Science, 75, H182-H189. https://doi.org/10.1111/j.1750-3841.2010.01675.x

[13] Syu, K.Y., Lin, C.L., Huang, H.C. and Lin, J.K. (2008) Determination of Theanine, GABA, and Other Amino Acids in Green, Oolong, Black, and Pu-Erh Teas with 
Dabsylation and High-Performance Liquid Chromatography. Journal of Agricultural and Food Chemistry, 56, 7637-7643. https://doi.org/10.1021/jf801795m

[14] Sava, V.M., Yang, S.-M., Hong, M.-Y., Yang, P.-C. and Huang, G.S. (2001) Isolation and Characterization of Melanic Pigments Derived from Tea and Tea Polyphenols. Food Chemistry, 73, 177-184. https://doi.org/10.1016/S0308-8146(00)00258-2

[15] Liang, Y., Zhang, L. and Lu, J. (2005) A Study on Chemical Estimation of Pu-Erh Tea Quality. Journal of the Science of Food and Agriculture, 85, 381-390. https://doi.org/10.1002/jsfa.1857

[16] Niwa, Y., Ishimoto, K. and Kanoh, T. (1990) Induction of Superoxide Dismutase in Leukocytes by Paraquat: Correlation with Age and Possible Predictor of Longevity. Blood, 76, 835-841.

[17] Han, L.K., Takaku, T., Li, J., Kimura, Y. and Okuda, H. (1999) Anti-Obesity Action of Oolong Tea. International Journal of Obesity, 23, 98-105. https://doi.org/10.1038/sj.ijo.0800766

[18] Zheng, G., Sayama, K., Okubo, T., Juneja, L.R. and Oguni, I. (2004) Anti-Obesity Effects of Three Major Components of Green Tea, Catechins, Caffeine and Theanine, in Mice. In Vivo, 18, 55-62.

[19] Lin, J.K. and Lin-Shiau, S.Y. (2006) Mechanisms of Hypolipidemic and Anti-Obesity Effects of Tea and Tea Polyphenols. Molecular Nutrition \& Food Research, 50, 211-217. https://doi.org/10.1002/mnfr.200500138

[20] Oi, Y., Hou, I.C., Fujita, H. and Yazawa, K. (2012) Antiobesity Effects of Chinese Black Tea (Pu-Erh Tea) Extract and Gallic Acid. Phytotherapy Research, 26, 475-481. https://doi.org/10.1002/ptr.3602

[21] Hou, Y., Shao, W., Xiao, R., Xu, K., Ma, Z., Johnstone, B.H. and Du, Y. (2009) $\mathrm{Pu}$-Erh Tea Aqueous Extracts Lower Atherosclerotic Risk Factors in a Rat Hyperlipidemia Model. Experimental Gerontology, 44, 434-439. https://doi.org/10.1016/j.exger.2009.03.007

[22] Valko, M., Leibfritz, D., Moncol, J., Cronin, M.T., Mazur, M. and Telser, J. (2007) Free Radicals and Antioxidants in Normal Physiological Functions and Human Disease. The International Journal of Biochemistry \& Cell Biology, 39, 44-84. https://doi.org/10.1016/j.biocel.2006.07.001 